\title{
IMPACTO DE LOS INCENDIOS SOBRE LA DIVERSIDAD VEGETAL, SIERRAS DE CORDOBA, ARGENTINA.
}

\section{IMPACT OF FIRES ON PLANT DIVERSITY, SIERRAS OF CÓRDOBA, ARGENTINA.}

\author{
Graciela Verzino ${ }^{1}$, Jacqueline Joseau ${ }^{2}$, Mónica Dorado ${ }^{3}$, Edgardo Gellert ${ }^{4}$, Sandra Rodríguez Reartes ${ }^{5}$ y Raúl \\ Nóbile $^{6}$
}

\section{Resumen}

Si bien se conoce que los incendios de campos ejercen impacto negativo sobre el bosque serrano, no existen antecedentes de trabajos que cuantifiquen la incidencia de los incendios sobre la diversidad vegetal de la región. El objetivo de este trabajo fue determinar el efecto de los incendios forestales sobre la composición florística, la estructura y la diversidad de la vegetación del Bosque Serrano en la provincia de Córdoba. El área de estudio se localizó sobre la ladera occidental de las Sierras Chicas de Córdoba. Se seleccionaron tres bosques quemados de distinta antigüedad, adyacentes a bosques sin quemar de características similares donde se aplicó un diseño de grupo de experimentos con cuatro repeticiones. En cada parcela se midieron árboles adultos y regeneración, cobertura y espesor del mantillo. Si bien la riqueza de especies arbóreas no se observó modificada por el incendio, la de arbustivas fue sustancialmente menor aún nueve años después de ocurrido el evento y las herbáceas aumentaron al año siguiente del incendio pero luego se equilibraron con el bosque testigo. La estructura vertical del bosque fue completamente alterada al desaparecer o reducirse drásticamente el estrato arbóreo. La abundancia de la regeneración de las especies arbóreas y arbustivas, así como su diversidad, resultaron menores en los sitios incendiados. Estos resultados permitieron cuantificar el grado de alteración de la diversidad y la estructura del Bosque Serrano a causa de los incendios, la que se mantiene nueve años después de ocurrido el siniestro.

Palabras clave: incendios forestales, biodiversidad, regeneración, Chaco Serrano, composición florística, estructura.

\begin{abstract}
Summary
Although it is well known that forest fires exert a negative impact upon forests in the Sierras de Córdoba, there is a lack of information regarding impact quantification which could help to assess the effect of fires on plant composition, structure and diversity. The objective of this work was to determine the effect of forest fires on plant composition, structure and diversity in the Bosque Serrano, Córdoba province. The study area was located on the west hillside of the Sierras Chicas, Córdoba. Three forests which had burned at different times were selected, adjacent to non-burned controls, using a group of experiments designed with 4 replicates. Adult trees and shrubs were measured, as well as regeneration, cover distribution and litter thickness. Although adult trees richness was not modified by fires, shrubs richness was substantially reduced even nine years after the event and herbs richness grew immediately after the fire but afterwards leveled with the control forest. The forests vertical structure was completely disturbed due to the dramatic reduction of the arboreal stratum. Abundance in the trees and shrubs regeneration, as well as diversity, were significantly lower in burned sites. These results allow us to quantify the degree of biodiversity and structure alteration in the Bosque Serrano due to forest fires, which remains even nine years after the event.
\end{abstract}

Key-words: forest fires, biodiversity, regeneration, Chaco Serrano, plant composition, structure.

\section{Introducción}

Las laderas de las sierras de Córdoba presentan, entre los 500 y $1300 \mathrm{~m}$ de altitud, una vegetación arbórea de particular fisonomía: el Bosque Serrano, componente orográfico de la región fitogeográfica del Parque Chaqueño.

El Bosque Serrano ha sufrido en los últimos cincuenta años, una notable reducción en la superficie total y en su riqueza florística (Luti et al., 1979). Las actividades extractivas de madera, leña, minerales, hierbas medicinales, aromáticas y fauna, conjuntamente con el sobrepastoreo, los incendios de campos, provocados o accidentales y el turismo han conducido a un deterioro marcado de la vegetación original, contribuyendo a modelar el paisaje actual 
(Programa MAB-UNESCO, 1986; Cabido \& Zak, 1999).

Entre todos los factores mencionados los incendios son quizás, los que inciden más profundamente sobre el ambiente de sierras, al actuar con marcada intensidad sobre los componentes del sistema y sus relaciones. Las Sierras del norte y noroeste se incluyen entre las áreas de mayor ocurrencia de incendios de la provincia. Allí, el fuego es utilizado como herramienta de manejo de pastizales sobrepastoreados los que, consecuentemente, son invadidos por especies herbáceas y arbustivas no deseables (Abril \& González, 1999). En las Sierras de Comechingones, al suroeste de la provincia de Córdoba, Beguet et al., (1989) observaron que áreas quemadas periódicamente, a medida que transcurre el tiempo, se van haciendo florísticamente más disímiles de las áreas sin quemar. En otras zonas del país se dan situaciones similares. Así, las quemas repetidas en un matorral de Larrea divaricata Cav., provocaron una pérdida importante de especies, cambios en la estructura y composición florística y en la capacidad de sustento del sistema natural (Martínez Carretero, 1995). Igualmente, en la Reserva Natural Villavicencio (Mendoza, Argentina), los incendios son responsables de cambios sustanciales en la composición florística y en la fisonomía del paisaje original así como de la pérdida de especies vegetales (Dalmaso et al., 1999). Sin embargo, los estudios realizados por Gobbi (1991) en bosques puros de Austrocedrus chilensis (D.Don) Florin Boutleje, no mostraron cambios significativos en la diversidad como consecuencia del fuego sobre la regeneración de los estratos arbustivo y herbáceo. En los bosques mediterráneos, Trabaud (1998) observó que la riqueza florística aumentaba tras el incendio alcanzando los valores máximos entre el primero y tercer año (debido sobre todo a las especies anuales que tendían a ocupar los espacios incendiados). A lo largo de los dos a tres años siguientes el número de especies disminuía progresivamente y a partir del quinto año la riqueza tendía a estabilizarse. Tampoco se encontraron diferencias importantes en diversidad y riqueza de especies en comparación con las áreas quemadas después de 30 años de protección en el área protegida de Los Llanos, Venezuela (Medina \& Bilbao, 1991).

Si bien se conoce que los incendios de campos ejercen un impacto sobre el bosque serrano, no existe una cuantificación de dicho impacto que permita valorar la incidencia de estos eventos sobre la biodiversidad vegetal de la región. Los cambios en los parámetros estructurales, así como en la riqueza y diversidad de la comunidad boscosa han mostrado ser valiosas herramientas para medir el impacto de un evento sobre la misma. El objetivo de este trabajo fue determinar el efecto de los incendios forestales sobre la composición florística, la estructura y la diversidad de la vegetación del Bosque Serrano en la provincia de Córdoba.

\section{Materiales y Métodos}

El área de estudio se localizó en el Bosque Serrano ubicado sobre la ladera occidental de las Sierras Chicas de Córdoba, aproximadamente a $30^{\circ} 50^{\prime}$ de latitud Sur y $64^{\circ} 30^{\prime}$ de longitud Oeste, en las inmediaciones de la localidad de Capilla del Monte, sobre el faldeo de los cerros Las Gemelas y Uritorco.

Para el estudio se seleccionaron tres bosques quemados de distinta antigüedad, adyacentes a bosques sin quemar de características similares en cuanto a altitud sobre el nivel del mar, exposición, composición específica, y estructura.

Se adoptó un diseño de grupo de experimentos con cuatro repeticiones sin restricciones a la aleatorización. La estructura de tratamientos fue un factorial $3 \times 2$, donde el primer factor fue la antigüedad desde el incendio, con tres niveles: Antigüedad $1: 1$ año desde el incendio, Antigüedad 2 : 2 años desde el incendio, Antigüedad 3:9 años desde el incendio; y el segundo factor la ocurrencia de incendios, con dos niveles: quemado y sin quemar (testigo). Dentro de cada uno de los 6 tratamientos resultantes se efectuaron muestreos sistemáticos delimitándose 4 parcelas permanentes (repeticiones) rectangulares de $20 \mathrm{~m}$ por $10 \mathrm{~m}$ de lado $\left(200 \mathrm{~m}^{2}\right)$ para la medición de los árboles y arbustos adultos (DAP $\geq$ $10 \mathrm{~cm})$. El recuento de renovales y plántulas forestales (DAP $<10 \mathrm{~cm}$ ) se efectuó en subparcelas de $10 \mathrm{~m}$ por $10 \mathrm{~m}$ de lado, ubicados en las cabeceras de las parcelas principales. La cobertura vegetal se determinó mediante el método de líneas interceptadas (Mateucci \& Colma, 1982) sobre los últimos $10 \mathrm{~m}$ de la parcela principal. Dado que la reducción en el espesor de mantillo es una de los principales consecuencias de los incendios de campo (Steinaker et al., 1999; Fisher et al., 1999) se midió, dentro de cada parcela principal, el espesor del mantillo a través de 8 mediciones al azar.

\section{Análisis de la información:}

Se efectuaron análisis descriptivos y gráficos con el objeto de visualizar el comportamiento general de las variables. Se estimaron los siguientes parámetros de la población:

Riqueza florística: Parámetro que refleja la presencia o ausencia de especies en una comunidad vegetal. Se enumeraron todas las espermatófitas y pteridófitas presentes en las parcelas, agrupadas por estratos (arbóreo, arbustivo y herbáceo) y discriminadas según antigüedad y ocurrencia del incendio. La mayoría de los individuos se identificaron hasta nivel de especie, y unos pocos hasta nivel de género o familia.

Cobertura vegetal: Es la proporción de terreno ocupado por la proyección perpendicular de las partes 
aéreas de los individuos considerados (Mateucci \& Colma, 1982). Se expresó en porcentaje de la superficie total.

En leñosas adultas:

Parámetros estructurales: $\mathrm{Se}$ determinaron abundancia absoluta y relativa y frecuencia absoluta y relativa de las especies leñosas arbóreas y arbustivas más importantes, siguiendo la metodología de Braun Blanquet (1979).

Diversidad: Se estimó la diversidad vegetal del bosque sin quemar mediante el índice de ShanonWeaver, como valor único que combina riqueza específica y equitatividad (Somarriba, 1999), aplicado sobre los ejemplares adultos de especies leñosas arbóreas y arbustivas.

En la regeneración:

Parámetros estructurales: $\mathrm{Se}$ determinaron abundancia absoluta y relativa y frecuencia absoluta y relativa de la regeneración de las especies leñosas arbóreas y arbustivas más importantes, siguiendo la metodología de Braun Blanquet (1979). Se analizó la distribución de la regeneración según su origen (semilla o rebrote de cepa) y su altura.

Diversidad: Se comparó la diversidad vegetal del bosque quemado con la del bosque sin quemar utilizando como variable el índice de Shanon-Weaver aplicado sobre la regeneración de especies leñosas arbóreas y arbustivas. Para ello se utilizó análisis de la varianza de acuerdo al modelo:

$$
y_{i j k}=\mu+\tau_{i}+\beta_{j}+\gamma_{k}+(\tau \beta)_{i j}+\varepsilon_{i j k}
$$

donde $y_{\mathrm{ijk}}=$ es el (ijk) ésimo valor de ISW; $\mu=$ es la media general, común a todos los tratamientos, $\tau_{\mathrm{i}=}$ efecto del i-ésimo nivel del factor antigüedad desde el incendio; $\beta_{\mathrm{j}}=$ efecto del $\mathrm{j}$-ésimo nivel del factor ocurrencia de incendios; $\gamma_{\mathrm{k}}=$ efecto de la k-ésima parcela, $(\tau \beta)_{\mathrm{ij}}=$ interacción de los niveles i y j de los factores $\tau$ y $\beta, \varepsilon_{\mathrm{ijk}=}$ error experimental asociado a la

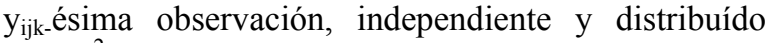
$\mathrm{N}\left(0, \sigma^{2}\right)$

Espesor de mantillo: Se estimó el espesor medio del mantillo en cada parcela de prueba.

\section{Resultados Y Discusión}

Riqueza florística: En el bosque testigo se registraron 93 especies, pertenecientes a 31 familias taxonómicas. De las especies presentes 11 fueron arbóreas-subarbóreas, 24 arbustivas y 58 herbáceas (Figura 1). En la zona de 1 año de antigüedad del incendio se hallaron 36 especies en el sitio quemado y 31 en el sitio sin quemar. En la zona de dos años de antigüedad, la riqueza florística fue de 39 especies en el sitio quemado y 43 especies en el sitio sin quemar. En la zona de nueve años de antigüedad del incendio se registraron 19 especies en el sitio quemado y 29 en el sitio sin quemar.

La riqueza de especies arbóreas fue similar en los sitios quemados y sin quemar (o testigo); mientras que la riqueza de arbustivas fue mayor en sitios testigo que en los quemados (Figura 1). Estos resultados, aparentemente contradictorios se explican por el carácter del parámetro analizado y por las características intrínsecas de las especies afectadas. La riqueza se mide por la presencia de especies distintas cualquiera sea su tamaño o edad. Así, la riqueza de los sitios testigo, con árboles adultos sin quemar, resulta similar a la de sitios incendiados con árboles adultos quemados y posteriormente rebrotados, porque se registran las especies arbóreas vivas, aunque sólo sea un rebrote. En cambio, la riqueza de arbustivas es menor en sitios con incendios recientes con respecto a los testigos porque algunas especies arbustivas más delicadas son destruidas totalmente por el fuego y tardan en reinstalarse. En cuanto a las herbáceas se observó mayor riqueza en los sitios recientemente quemados (1 y 2 años desde el incendio) que en el sitio con 9 años desde el incendio; esto debido a que las especies de ciclo anual responden más rápidamente y tienden a ocupar los claros producidos por el fuego (Donoso, 1981). Dicha dinámica guarda similitud con lo descripto por Prodon et al. (1984) y Trabaud (1998), para los bosques mediterráneos.

Cobertura: Los incendios afectaron en algunos casos hasta el 100\% del dosel arbóreo y arbustivo, situación que se mantuvo nueve años luego del evento. En la zona quemada de un año la cobertura de Lithrea molleoides "molle de beber" fue del $11 \% \mathrm{y}$ Schinopsis haenkeana "horco quebracho" el 4 \%. En el sitio testigo, Lithrea molleoides ocupó el $37 \%$ y Schinopsis haenkeana el $0,5 \%$. En el primero se observó una alta proporción de piedras $(11 \%)$ y suelo desnudo $(15 \%)$. El segundo mostró un alto porcentaje de "paja brava", Melica macra $(38.25 \%)$.

En la zona quemada de dos años se observó una importante presencia de Clematis montevidensis (26 $\%$ ) y $7 \%$ de suelo desnudo. En general el porcentaje de participación de las distintas especies en este sitio no alcanza al $10 \%$, incluyendo arbóreas y arbustivas, salvo "cabello de angel" Clematis montevidensis., "amor seco" Bidens pilosa L. (11\%) y "rama negra" Conyza bonariensis (11 \%), estas últimas consideradas malezas en campos de laboreo. En el sitio testigo se registró una cobertura arbórea constituida en un $47 \%$ por Lithrea molleoides y un 20 $\%$ de Schinopsis haenkeana, ausentes en el sitio quemado.

La zona quemada de 9 años presentó un bajo porcentaje de cobertura arbórea $(2 \%)$ con Lithrea molleoides., en tanto la zona no incendiada mostró una buena cobertura arbórea compuesta por Lithrea molleoides (26\%) y Schinopsis haenkeana. (18\%). Si bien no es significativa la diferencia en la riqueza florística entre sitio quemado y testigo (Figura 1en Apéndice), el estrato arbóreo no se ha recuperado aún nueve años después del incendio. En la zona quemada se observó un $7 \%$ de suelo desnudo mientras que en 
el testigo llegó al $4 \%$. Los valores de cobertura obtenidos ratifican que los incendios inciden negativamente sobre la cobertura arbórea, aumentando significativamente la proporción de suelo desnudo

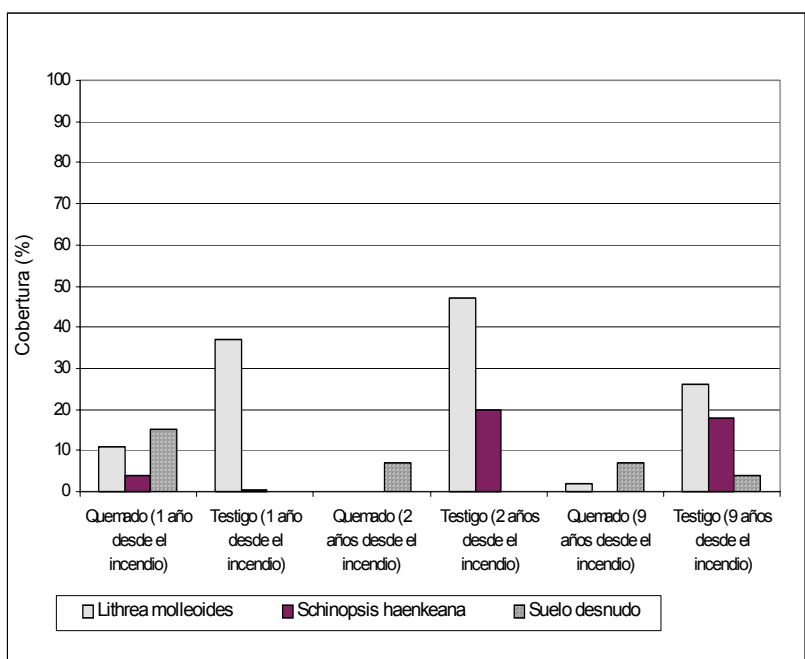

(Fisher et al., 1999) (Figura 2).

Figura 2. Cobertura de Lithrea molleoides (Vell.) Engl. , Schinopsis haenkeana Engl. y suelo desnudo en las tres zonas del estudio, en sitios quemados y testigos sin quemar.

\section{Análisis de leñosas adultas:}

Parámetros estructurales: Dada la destrucción casi total del estrato leñoso adulto en los bosques quemados se efectuó el análisis de los parámetros estructurales sólo en bosques sin quemar (testigos). En los tres sitios muestreados Lithrea molleoides fue la especie de mayor abundancia relativa y absoluta, y mayor frecuencia relativa y absoluta. Esta especie es la más representativa del Bosque Serrano (Luti et al., 1979). Otras especies registradas fueron Acacia caven "espinillo", A. atramentaria "espinillo negro" y Schinopsis haenkeana (Tabla 1).

Diversidad: El índice de diversidad de Shanon Weaver, obtenido sobre individuos adultos de árboles y arbustos en los bosques sin quemar fue de 1.3 en cerro Uritorco y 1.12 - 1.02 en las dos zonas en Las Gemelas. Este índice, utilizado mayormente en selvas tropicales y subtropicales, varía entre valores de 1.5 y 3.5 y raramente sobrepasa 4.5 (Magurrant, 1988; Somarriba, 1999). Los valores estimados en el presente estudio fueron menores que aquellos de selvas tropicales y subtropicales, confirmando los resultados de Wallace (1898), Dobzhansky (1950) y Fisher (1960) (citados por Mc Naughton \& Wolf, 1984), en el sentido de que los ecosistemas templados presentan comunidades mucho menos diversas que los tropicales.
Tabla 1. Parámetros estructurales de individuos adultos en bosques sin quemar, considerando las especies arbóreas y arbustivas más frecuentes.

Antigüedad 1 - Sin quemar

\begin{tabular}{lrrrrr}
\hline Especie & $\begin{array}{c}\text { Abunda } \\
\text { ncia }\end{array}$ & $\begin{array}{c}\text { Abundancia } \\
\text { absoluta }\end{array}$ & $\begin{array}{c}\text { Abundancia } \\
\text { Relativa }\end{array}$ & $\begin{array}{c}\text { Frecuencia } \\
\text { absoluta }\end{array}$ & $\begin{array}{r}\text { Frecuencia. } \\
\text { Relativa }\end{array}$ \\
\hline $\begin{array}{l}\text { Lithrea } \\
\text { molleoides }\end{array}$ & $\mathbf{2 2}$ & $\mathbf{2 7 5 . 0 0}$ & $\mathbf{3 5 . 1 1}$ & $\mathbf{1 0 0 . 0 0}$ & $\mathbf{3 3 . 3 3}$ \\
$\begin{array}{l}\text { Schinus } \\
\text { fasciculatus } \\
\text { Acacia sp }\end{array}$ & 1 & 50.00 & 6.38 & 25.00 & 8.33 \\
$\begin{array}{l}\text { Schinopsis } \\
\text { haenkeana }\end{array}$ & 2 & 100.00 & 12.77 & 25.00 & 8.33 \\
$\begin{array}{l}\text { Condalia } \\
\text { montana }\end{array}$ & 5 & 83.33 & 10.64 & 75.00 & 25.00 \\
$\begin{array}{l}\text { Kagenekia } \\
\text { lanceolata }\end{array}$ & 5 & 125.00 & 15.96 & 50.00 & 16.67 \\
TOTALES & 3 & 150.00 & 19.15 & 25.00 & 8.33 \\
\hline & 38 & 783.33 & 100.00 & & 100.00 \\
\hline
\end{tabular}

Antigüedad 2 - Sin quemar

\begin{tabular}{|c|c|c|c|c|c|}
\hline Especie & $\begin{array}{c}\text { Abunda } \\
\text { ncia }\end{array}$ & $\begin{array}{c}\text { Abundancia } \\
\text { absoluta }\end{array}$ & $\begin{array}{c}\text { Abundancia } \\
\text { Relativa }\end{array}$ & $\begin{array}{c}\text { Frecuencia } \\
\text { absoluta }\end{array}$ & $\begin{array}{c}\text { Frecuencia. } \\
\text { Relativa }\end{array}$ \\
\hline $\begin{array}{l}\text { Lithrea } \\
\text { molleoides }\end{array}$ & 28 & 350.00 & 40.58 & 100.00 & 30.77 \\
\hline Acacia caven & 1 & 50.00 & 5.80 & 25.00 & 7.69 \\
\hline Acacia $s p$ & 19 & 237.50 & 27.54 & 100.00 & 30.77 \\
\hline $\begin{array}{l}\text { Schinopsis } \\
\text { haenkeana }\end{array}$ & 18 & 225.00 & 26.09 & 100.00 & 30.77 \\
\hline TOTALES & 66 & 862.50 & 100.00 & & 100.00 \\
\hline
\end{tabular}

Antigüedad 3 - Sin quemar

\begin{tabular}{lrrrrr}
\hline \multicolumn{1}{c}{ Especie } & $\begin{array}{c}\text { Abunda } \\
\text { ncia }\end{array}$ & $\begin{array}{c}\text { Abundancia } \\
\text { absoluta }\end{array}$ & $\begin{array}{c}\text { Abundancia } \\
\text { Relativa }\end{array}$ & $\begin{array}{c}\text { Frecuencia } \\
\text { absoluta }\end{array}$ & $\begin{array}{c}\text { Frecuencia. } \\
\text { Relativa }\end{array}$ \\
\hline $\begin{array}{l}\text { Lithrea } \\
\text { molleoides }\end{array}$ & $\mathbf{1 9}$ & $\mathbf{2 3 7 . 5 0}$ & $\mathbf{5 1 . 3 5}$ & $\mathbf{1 0 0 . 0 0}$ & $\mathbf{3 3 . 3 3}$ \\
$\begin{array}{l}\text { Acacia caven } \\
\begin{array}{l}\text { Schinopsis } \\
\text { haenkeana }\end{array}\end{array}$ & 6 & 75.00 & 16.22 & 100.00 & 33.33 \\
$\begin{array}{l}\text { Ximenia } \\
\text { americana }\end{array}$ & 1 & 50.00 & 10.81 & 25.00 & 8.33 \\
$\begin{array}{l}\text { Acacia aroma } \\
\text { TOTALES }\end{array}$ & 1 & 50.00 & 10.81 & 50.00 & 16.67 \\
& 29 & 462.50 & 100.00 & & 8.33 \\
\end{tabular}

Referencias:

Abundancia: Número de individuos de una especie registrados en la muestra.

Abundancia absoluta: número de individuos de una especie por unidad de superficie, en individuos/ha. Abundancia relativa: relación entre el número de individuos de una especie y el número de individuos totales por unidad de superficie, en \%. Frecuencia absoluta: ocurrencia de una especie en una unidad experimental en relación al número total de unidades experimentales, en $\%$. Frecuencia relativa: relación de las frecuencias absolutas de la iésima especie respecto al total de las frecuencias absolutas, en $\%$.

\section{Análisis de la Regeneración:}

Parámetros estructurales: Los bosques sin quemar presentaron abundante regeneración de leñosas arbóreas y arbustivas, destacándose: Lithrea molleoides, "durazno del campo" Kageneckia 
lanceolata y Schinopsis haenkeana. La abundancia absoluta de la regeneración en sitios incendiados fue, en promedio, la mitad de la de los sitios testigos. En la zona con incendio más reciente (1 año) fue escasa, mientras que en las zonas de 2 y 9 años desde el incendio la regeneración de los "espinillos" (Acacia caven y Acacia atramentaria) y de la "chilca" (Flourensia campestris.) fue importante, dando muestras de su adaptación a los incendios (Tabla 2). Cabido \& Zak (1999) observaron un comportamiento similar de estas especies frente a los incendios.

Tabla 2. Abundancia absoluta de la regeneración de árboles y arbustos más frecuentes.

\begin{tabular}{|c|c|c|c|c|c|c|}
\hline \multirow[t]{2}{*}{ Especies } & \multicolumn{2}{|c|}{ Antigüedad 1} & \multicolumn{2}{|c|}{ Antigüedad 2} & \multicolumn{2}{|c|}{ Antigüedad 3} \\
\hline & Quemado & Testigo & Quemado & Testigo & Quemado & Testigo \\
\hline & 100.00 & 200.00 & 1650.00 & 533.33 & 925.00 & 433.33 \\
\hline \multicolumn{7}{|l|}{ Acacia spp* } \\
\hline Celtis spp** & & & 200.00 & 500.00 & 200.00 & 375.00 \\
\hline $\begin{array}{l}\text { Condalia } \\
\text { spp*** }\end{array}$ & 650.00 & 650.00 & 600.00 & 300.00 & & 200.00 \\
\hline Fagara coco & 200.00 & & 100.00 & & & \\
\hline $\begin{array}{l}\text { Flourensia } \\
\text { campestris }\end{array}$ & 196.87 & 201.60 & & 73.33 & 890.00 & 530.00 \\
\hline $\begin{array}{l}\text { Jodina } \\
\text { rhombifolia }\end{array}$ & & 200.00 & & & & \\
\hline $\begin{array}{l}\text { Kageneckia } \\
\text { lanceolata }\end{array}$ & 233.33 & 1600.00 & & & & 2750.00 \\
\hline $\begin{array}{l}\text { Lithrea } \\
\text { molleoides }\end{array}$ & 200.00 & 200.00 & 100.00 & 1725.00 & 266.67 & 1350.00 \\
\hline $\begin{array}{l}\text { Maytenus } \\
\text { spinosa }\end{array}$ & & 100.00 & 100.00 & & & 100.00 \\
\hline $\begin{array}{l}\text { Ruprechtia } \\
\text { apetala }\end{array}$ & 433.33 & 750.00 & & & & \\
\hline $\begin{array}{l}\text { Schinopsis } \\
\text { haenkeana }\end{array}$ & 100.00 & 825.00 & & 950.00 & 133.33 & 600.00 \\
\hline $\begin{array}{l}\text { Schinus } \\
\text { fasciculatus }\end{array}$ & 100.00 & 475.00 & & 375.00 & 350.00 & 366.67 \\
\hline \multirow[t]{2}{*}{$\begin{array}{l}\text { Ximenia } \\
\text { americana }\end{array}$} & & & & 200.00 & & \\
\hline & 2213.53 & 5201.60 & 3225.00 & 4656.66 & 2765.00 & 6705.00 \\
\hline Tota & & & & & & \\
\hline
\end{tabular}

*A.caven y A.atramentaria $\quad{ }^{* *}$ Celtis tala y C. pallida ${ }^{* * *}$ Condalia microphylla y $C$. montana

Referencias: Antigüedad 1: Incendio 1 año;

Antigüedad 2: Incendio 2 años;

Antigüedad 3: Incendio 9 años

La regeneración se agrupó en tres categorías según su origen (por semilla o por rebrote de la base) (Figura 3 en Anexo), lo que permitió visualizar regeneración por semilla significativamente mayor en los sitios testigo con respecto a los quemados. En los sitios quemados, la regeneración fue predominantemente por rebrote de la base en las zonas de 1 y 2 años de antigüedad del incendio, o indistintamente por rebrote de la base y semillas en la zona de 9 años desde el incendio. Se manifiestan aquí estrategias de sobrevivencia basadas principalmente en la multiplicación vegetativa, ampliamente difundidas en zonas con incendios recurrentes (Trabaud, 1992, 1998; Vallejo \& Alloza, 1998) o en zonas áridas, con condiciones poco favorables para la regeneración por semillas (Moglia \& Jofré, 1998) En este caso puede observarse que las especies arbóreas y arbustivas más importantes del Chaco Serrano se regeneran por rebrotes de yemas en la base de los troncos. En cuanto a su altura, en general se observó un mayor número de individuos en los sitios testigo, cualquiera fuera la categoría de altura, salvo en la zona de 2 años desde el incendio, donde hubo más rebrotes mayores de $50 \mathrm{~cm}$ de altura en los sitios quemados que en los testigo.

Diversidad de especies: El análisis de la varianza, utilizado para comparar los ISW de la regeneración de todas las parcelas de la muestra, permitió detectar diferencias significativas entre la diversidad de los bosques quemados y los bosques testigo (Tabla 3). En general, la diversidad de los bosques testigo fue mayor que la de los bosques quemados, no resultando significativa la diferencia puesta de manifiesto por el gráfico en la zona de 1 año desde el incendio (Figura 4). Estos resultados coinciden con los de Martínez Carretero (1995) y los de Dalmaso et al. (1999). El primero señala una pérdida importante de especies, cambios en la estructura y composición florística y en la capacidad de sustento del sistema natural debido a quemas repetidas en un matorral de Larrea divaricata, mientras que el segundo describe similares consecuencias como resultado de incendios en la Reserva Natural Villavicencio (Mendoza, Argentina). Asimismo, se detectaron diferencias entre la zona de 1 año de antigüedad del incendio (falda del cerro Uritorco), por un lado, y las zonas de 2 y 9 años de antigüedad del incendio, en Las Gemelas, por el otro, atribuible a condiciones ambientales levemente distintas y a distintas características del incendio (intensidad y duración), que pueden afectar en forma diferencial la capacidad de recuperación del ecosistema (Hodgson \& Heislers, 1972).

Espesor de mantillo: La proporción de suelo desnudo alcanzó a $15 \%$ en el sitio quemado más recientemente. Los suelos de escaso espesor y poco desarrollo de las Sierras de Córdoba al perder la vegetación por acción del fuego recurrente, quedan expuestos a insolación, acción del viento y escorrentía, ampliando las condiciones mecánicas de degradación de las pendientes lo que profundiza la destrucción del bosque serrano (Herrera et al., 1978; Beguet et al., 1987). El mantillo, al cabo de 1 y 2 años del incendio, fue cinco veces más delgado que en los bosques testigos (Figura 5). Aquí, también, como lo manifestaran Steinaker et al. (1999) el espesor del mantillo resulta ser una variable valiosa para estimar el impacto del incendio sobre el sistema natural. 
Tabla 3. Análisis de la varianza del Indice de Shannon Weaver de cada parcela de la muestra.

\section{Análisis de la varianza}

\begin{tabular}{ccccc} 
Variable & $\mathrm{N}$ & $\mathrm{R}^{2}$ & $\mathrm{R}^{2} \mathrm{Aj}$ & $\mathrm{CV}$ \\
\hline indiceshannon & 23 & 0.60 & 0.44 & 18.07 \\
\hline
\end{tabular}

Datos desbalanceados en celdas.

Cuadro de Análisis de la Varianza (SC tipo I)

\begin{tabular}{lccccc} 
F.V. & SC & gl & CM & F & p-valor \\
\hline Modelo & 1.17 & 6 & 0.19 & 3.93 & 0.0133 \\
zona & 0.50 & 2 & 0.25 & 5.01 & 0.0204 \\
sitio & 0.32 & 1 & 0.32 & 6.35 & 0.0227 \\
parcela & 0.36 & 3 & 0.12 & 2.40 & 0.1062 \\
Error & 0.79 & 16 & 0.05 & & \\
Total & 1.96 & 22 & & & \\
\hline
\end{tabular}

Test:Tukey Alfa: $=\mathbf{0 . 0 5}$ DMS: $=\mathbf{0 . 2 9 3 8 4}$

Error: $0.0496 \mathrm{gl}: 16$

$\begin{array}{lcccc}\text { zona } & \text { Medias } & \mathrm{n} & & \\ 1.00 & 1.10 & 8 & \mathrm{~A} & \\ 2.00 & 1.17 & 8 & \mathrm{~A} & \mathrm{~B} \\ 3.00 & 1.45 & 7 & & \text { B }\end{array}$

Letras distintas indican diferencias significativas $(p<=0.05)$

Test:Tukey Alfa: $=\mathbf{0 . 0 5}$ DMS: $=\mathbf{0 . 1 9 7 0 2}$

Error: $0.0496 \mathrm{gl}: 16$

sitio Medias $\mathrm{n}$

$\begin{array}{lllll}1.00 & 1.10 & 11 & \mathrm{~A} & \\ 2.00 & 1.35 & 12 & & \mathrm{~B}\end{array}$

Letras distintas indican diferencias significativas $(p<=0.05)$

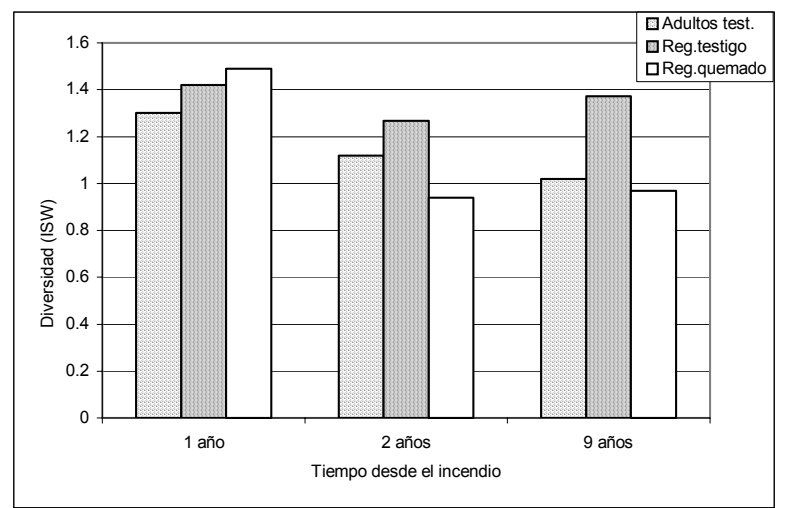

Figura 4. Diversidad, expresada por el Índice de Shannon-Weaver, en tres zonas del Bosque Serrano, considerando individuos adultos del bosque testigo, regeneración del bosque testigo y regeneración del bosque quemado.

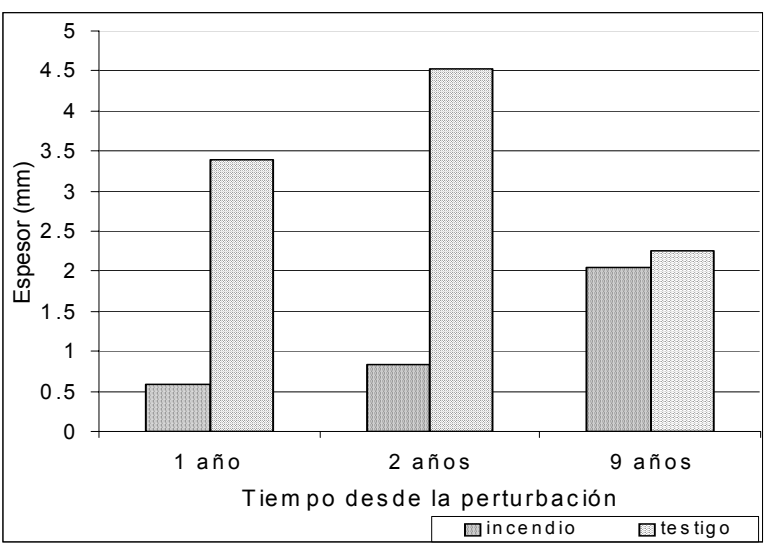

Figura 5. Espesor promedio del mantillo en tres zonas con 1, 2 y 9 años desde la ocurrencia del incendio, respectivamente. Las barras a la derecha representan el espesor de mantillo en los sitios testigo ( $\sin$ quemar).

\section{Conclusiones}

Si bien la riqueza de especies arbóreas no se vio modificada por el incendio, la de arbustivas fue sustancialmente menor aún 9 años después de ocurrido el evento y las herbáceas aumentaron al año siguiente del incendio pero luego se equilibraron con el bosque testigo. La estructura vertical del bosque se vio completamente alterada al desaparecer el estrato arbóreo en dos de las zonas estudiadas (Las Gemelas) y al reducirse marcadamente en la tercer zona (Uritorco). La abundancia de la regeneración de las especies arbóreas y arbustivas más frecuentes, así como su diversidad, resultaron significativamente menor en los sitios incendiados. Estos resultados permitieron cuantificar el grado de alteración de la diversidad y la estructura de la vegetación del Bosque Serrano a causa de los incendios forestales, la que se mantiene, en este caso, hasta nueve años después de ocurrido el siniestro. Asimismo, contribuirán a valorar la incidencia de los incendios sobre las funciones de producción, protección y estéticas de estos bosques.

Agradecimientos: El presente proyecto se realizó con fondos provistos por la Secretaría de Ciencia y Tecnología de la Universidad Nacional de Córdoba. Los autores agradecen al Complejo Turístico Agua de los Palos, donde se instalaron algunas parcelas de muestreo, a la Municipalidad de Capilla del Monte, a la Asociación Bomberos Voluntarios de Capilla del Monte y a la Asociación Rumbos, especialmente a Esteban "Tato" Espinosa, por el valioso apoyo brindado para la selección y muestreo de los sitios. A la Dra María del Pilar Díaz, por su contribución en el diseño del experimento. A todos los estudiantes de Silvicultura que participaron en el muestreo y procesamiento de la información. 


\section{Literatura citada}

Abril A. \& González C. 1999. Dinámica de la fertilidad y de las poblaciones microbianas en suelos afectados por incendios en las Sierras de Córdoba (Argentina). Agriscientia. Vol XVI: 63-70.

Beguet H., D’Andrea S. \& Montoni N. 1987. Influencia del fuego en un pastizal natural de las Sierras de Comechingones. Primeras Jornadas Nacionales de Zonas Áridas y Semiáridas. Santiago del Estero.: 332-333.

Braun Blanquet J. 1979. Fitosociología. Bases para el estudio de las comunidades vegetales. H. Blume. Ed, Madrid, España.

Cabido M. \& Zak M. 1999. Vegetación del norte de Córdoba. SAGyRR, Córdoba.

Dalmasso A., Martínez Carretero E., Videla F., Puig S. \& Candia R. 1999. Reserva Natural Villavicencio (Mendoza, Argentina). Plan de Manejo. Multequina. 8: 11-50.

Donoso Z. 1981. Ecología forestal. El bosque y su medio ambiente. Ed. Univ. Chile.

Fisher S., Madanes N.; Vicari R. \& Bonaventura S.M. 1999. Análisis de los atributos estructurales de un espartillar disturbado por fuego (Reserva estricta Otamendi). XIX Reunión Argentina de Ecología.: 99

Gobbi M. 1991. Regeneración post-incendio del sotobosque de cipresales. Resúmenes XXIII Jornadas Argentinas de Botánica. Bariloche. : 270.

Herrera M., Bertrán M., Galera F.M., Luti R. \& Menghi M. 1978. Incendio y pastoreo en estepas de altura de las Sierras Chicas de Córdoba. Ecología. 3: 95-99.

Hodgson A. \& Heislers A. 1972. Some aspects of the role of forest fire in South Eastern Australia. Actas del VII Congreso Forestal Mundial. Tomo II: 2858-2869.

Luti R., Bertrán de Solís M., Galera F.M., Muller de Ferreira N., Berzal M., Nores M., Herrera M. \& Barrera J.C. 1979. Vegetación. En: Geografía física de la provincia de Córdoba. Ed. Boldt, Bs. As.

Magurrant A.E. 1988. Ecological diversity and its measurements. Princeton University Press, Princeton New Jersey. USA.
Martínez Carretero E. 1995. Los incendios forestales en la Argentina. Multequina. 4: 105-114.

Matteucci S. \& Colma A. 1982. Metodología para el estudio de la vegetación. Monografía N 22. Ed. Eva Chesneau, Washington.

Mc Naughton S.J. \& Wolf L.L. 1984. Ecología General. Ed. Omega S.A., Barcelona.

Medina E. \& Bilbao B. 1996. Relaciones nutricionales e impacto de incendios sobre la producción de materia orgánica de pastizales naturales y cultivados. Biodiversidad y funcionamiento de pastizales y sabanas en América Latina. Ed. CYTED y CIELAT.

Moglia M. \& Jofré G. 1998. Respuestas al desmonte de las especies leñosas en un bosque de quebracho blanco (Aspidosperma quebracho-blanco Schletcht) y algarrobo (Prosopis flexuosa DC). Фhyton. Rev. Internac. de Botánica Experimental. 63 (1/2): 257-265

Prodon R., Fons R. \& Peter A.M. 1984. L'impact du feu sur la végétatiom, les oiseaux et les micromammifféres dans diverses formations méditerranéennes des pyrénées orientales: premiers résultats. Revue Ecologie Terre et Vie. 39: 129-158.

Programa MAB-UNESCO. 1986. Programa de Investigación Integrado y Entrenamiento en la región andina y montañas extra-andinas. UNESCO/MABPNUMA-CERNAR.

Somarriba E. 1999. Diversidad Shannon. Agroforestería en las Américas. 6 (23): 72-74.

Steinaker D.F., Aguilera M.O. \& Avila A.O. 1999. Respuesta de las especies del pastizal pampeano semiárido a una quema de otoño y primavera. XIX Reunión Argentina de Ecología. : 113.

Trabaud L. 1998. Recuperación y regeneración de ecosistemas mediterráneos incendiados. Serie Geográfica. Incendios forestales. 7: 37-47.

Vallejo R. \& Alloza J.A. 1998. The restoration of burned lands: The case of Eastern Spain. Large forest fires. Backbuys Publ., Leiden, The Netherlands. : 91-108. 


\section{ANEXOS}

Figura 1. Riqueza florística ordenada por estrato y discriminada según antigüedad y ocurrencia de los incendios. Referencias: Antigüedad 1: Incendio 1 año; Antigüedad 2: Incendio 2 años; Antigüedad 3: Incendio 9 años.

\begin{tabular}{|c|c|c|c|c|c|c|}
\hline Especie & Antig.1 & Antig. 2 & Antig. 3 & Antig.1 & Antig. 2 & Antig. 3 \\
\hline & \multicolumn{3}{|c|}{ Quemado } & \multicolumn{3}{|c|}{ Sin Quemar } \\
\hline ARBOREAS & & & & & & \\
\hline Acacia aroma Gillies ex Hook.\&Arn. & & & & & & \\
\hline Acacia atramentaria Benth. & & & & & & \\
\hline Acacia caven (Molina) Molina & & & & & & \\
\hline Acacia sp & & & & & & \\
\hline Celtis tala Gillies ex Planch. & & & & & & \\
\hline Fagara coco (Gillies)Engl. & & & & & & \\
\hline Jodina rhombifolia (Hook. \& Arn.) Reissek & & & & & & \\
\hline Lithrea molleoides (Vell.) Engl. & & & & & & \\
\hline Prosopis sp & & & & & & \\
\hline Ruprechtia apetala Wedd. & & & & & & \\
\hline Schinopsis haenkeana Engl. & & & & & & \\
\hline Suma parcial & 6 & 5 & 5 & 6 & 7 & 6 \\
\hline ARBUSTIVAS & & & & & & \\
\hline Aloysia gratissima (Gillies \& Hook.) Tronc. & & & & & & \\
\hline Baccharis articulata (Lam.) Pers. & & & & & & \\
\hline Baccharis flabellata Hook. \& Arm- & & & & & & \\
\hline Budleja cordobensis Griseb. & & & & & & \\
\hline Caesalpinia gilliesii (Wall. ex Hook.) D. Dietr. & & & & & & \\
\hline Celtis pallida Torr. & & & & & & \\
\hline Cestrum parqui L'Hér & & & & & & \\
\hline Colletia spinosissima J.F. Gmel. & & & & & & \\
\hline Condalia microphilla Cav. & & & & & & \\
\hline Condalia montana A. Cast. & & & & & & \\
\hline Croton sarcopetalus Muell. Arg. & & & & & & \\
\hline Dicotiledónea arbustiva & & & & & & \\
\hline Euforbiacea & & & & & & \\
\hline Eupatorium buniifolium Hook. \& Arn. & & & & & & \\
\hline Eupatorium sp & & & & & & \\
\hline Flourensia campestris Griseb. & & & & & & \\
\hline Kageneckia lanceolata Ruiz \& Pav. & & & & & & \\
\hline Lantana balansae Briq. f. & & & & & & \\
\hline Lippia sp & & & & & & \\
\hline Lippia turbinata Griseb. & & & & & & \\
\hline Maytenus spinosa (Griseb.) Lourteig \& O'Donell & & & & & & \\
\hline Schinus fasciculata (Griseb) I.M. Johnst. & & & & & & \\
\hline Schinus $s p$ & & & & & & \\
\hline Ximenia americana $\mathrm{L}$. & & & & & & \\
\hline Suma parcial & 11 & 9 & 7 & 13 & 14 & 13 \\
\hline HERBACEAS & & & & & & \\
\hline Abutilon sp & & & & & & \\
\hline Achyrocine tomentosa Rusby & & & & & & \\
\hline Amarantacea & & & & & & \\
\hline Asteracea & & & & & & \\
\hline Bidens pilosa $\mathrm{L}$. & & & & & & \\
\hline Briza sp & & & & & & \\
\hline Cactacea & & & & & & \\
\hline Caliceracea & & & & & & \\
\hline Clematis montevidensis Spreng. & & & & & & \\
\hline $\begin{array}{l}\text { Commelina virginica (L.)var angustifolia (Mich.) } \\
\text { C.B. Clarkee }\end{array}$ & & & & & & \\
\hline Conyza bonaerensis (L.) Cronquist & & & & & & \\
\hline Desmodium $s p$ & & & & & & \\
\hline Deuterocohnia longipetala (Baker) Mez & & & & & & \\
\hline
\end{tabular}




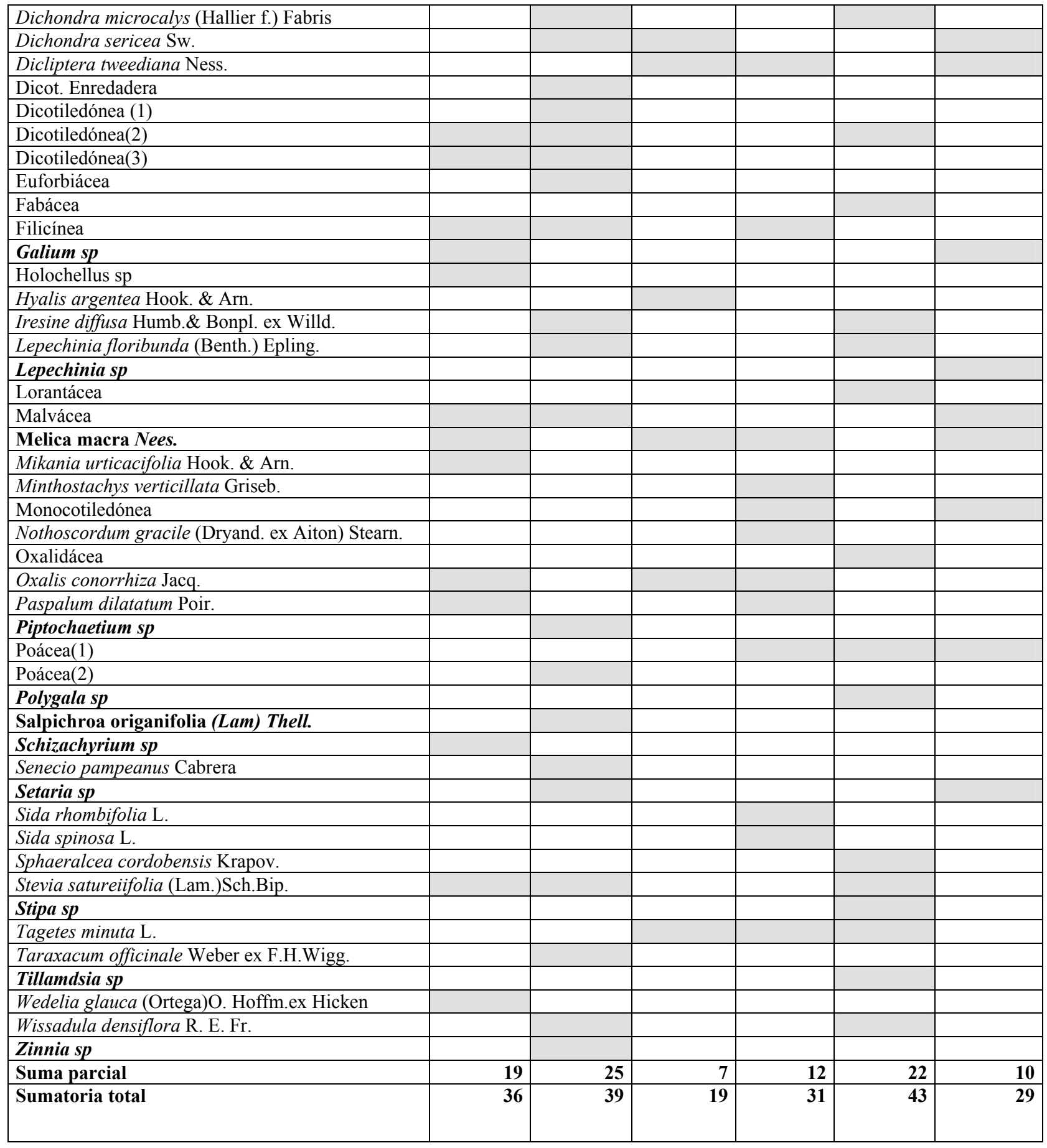


Figura 3. Regeneración de especies arbóreas y arbustivas, clasificada por tipo y por altura de la regeneración Referencias: Tipo de regeneración: 1: semilla, 2: rebrote de la base (- de 5 renuevos), 3: rebrote de la base ( + de 5 renuevos). Clase de altura: $\mathrm{a}: \leq 50 \mathrm{~cm}, \mathrm{~b}:>50 \mathrm{~cm}$ a $\leq 100 \mathrm{~cm}, \mathrm{c}:>100 \mathrm{~cm}$
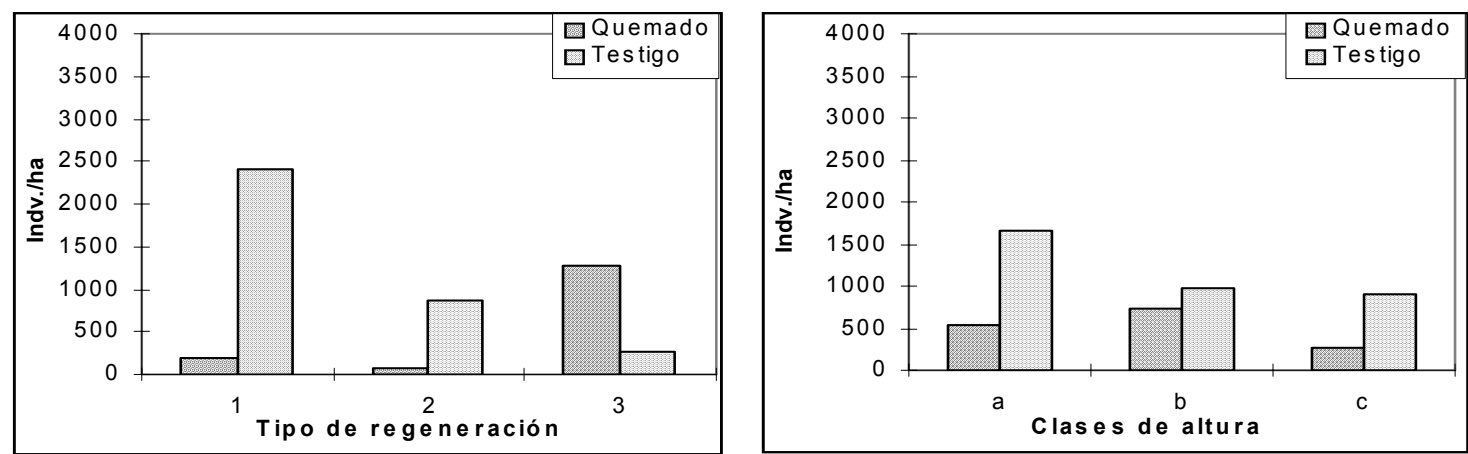

Zona 1: Incendio de 1 año, Uritorco
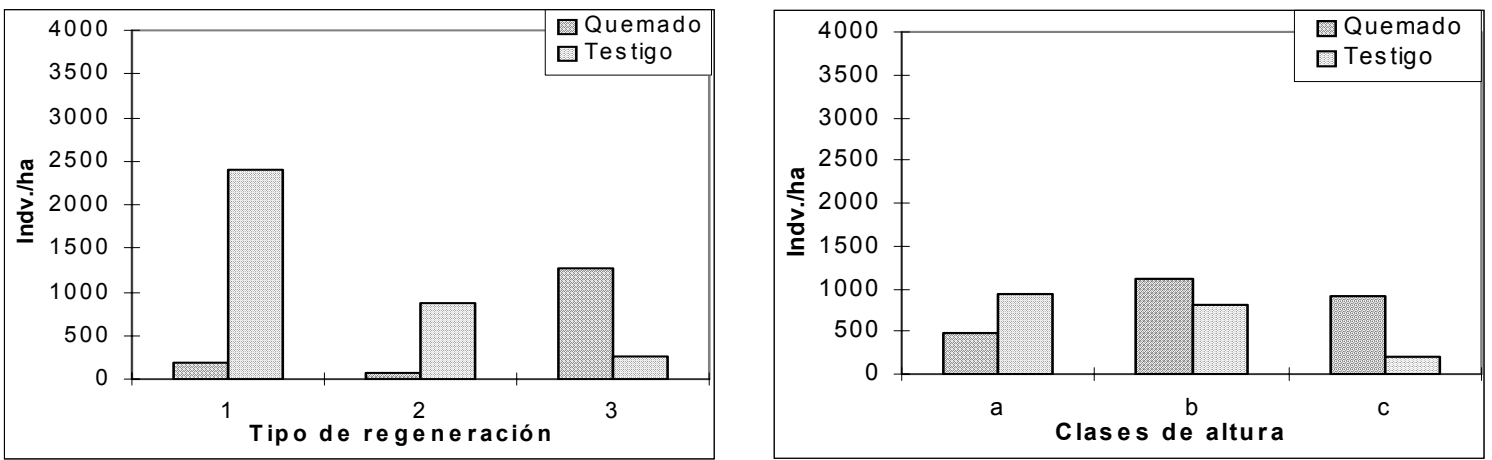

Zona 2 Incendio de 2 años, Las Gemelas
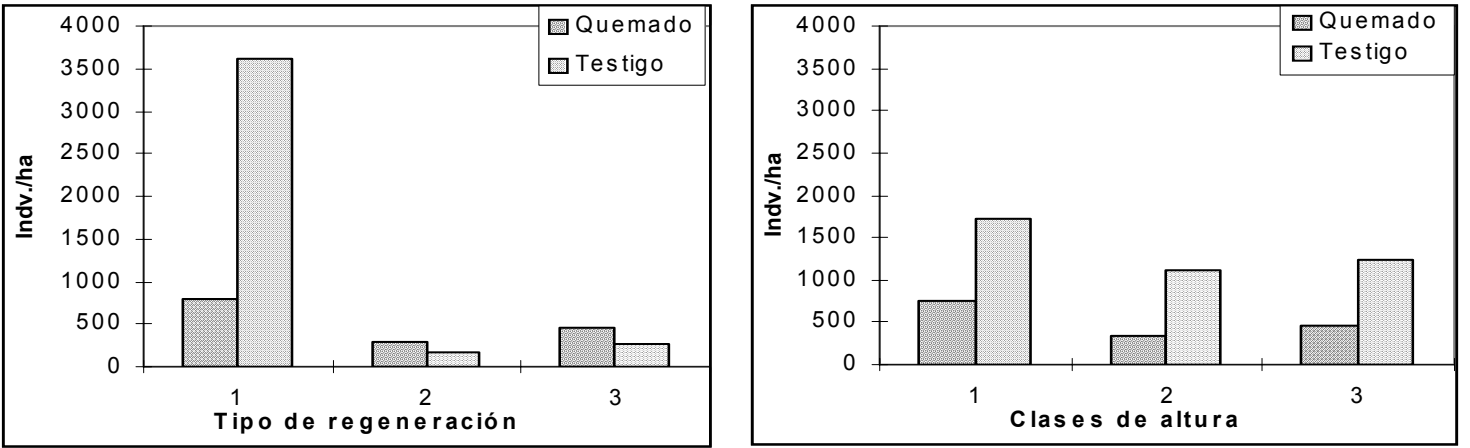

Zona 3: Incendio de 9 años, Las Gemelas

${ }^{1}$ : Silvicultura, Facultad de Ciencias Agropecuarias, Universidad Nacional de Córdoba. C.C. 509 - (5000) - Córdoba - ARGENTINA. gverzino@agro.uncor.edu

${ }^{2}$ Silvicultura, Facultad de Ciencias Agropecuarias, Universidad Nacional de Córdoba.

C.C. 509 - (5000) - Córdoba - ARGENTINA jajoseau@agro.uncor.edu

${ }^{3}$ Silvicultura, Facultad de Ciencias Agropecuarias, Universidad Nacional de Córdoba.

C.C. 509 - (5000) - Córdoba - ARGENTINA mdorado@agro.uncor.edu

${ }^{4}$ Silvicultura, Facultad de Ciencias Agropecuarias, Universidad Nacional de Córdoba.

C.C. 509 - (5000) - Córdoba - ARGENTINA ovejaedg@yahoo.com.ar

${ }^{5}$ Silvicultura, Facultad de Ciencias Agropecuarias, Universidad Nacional de Córdoba.

C.C. 509 - (5000) - Córdoba - ARGENTINA saroreartes@yahoo.com.ar

${ }^{6}$ Terapéutica Vegetal, Facultad de Ciencias Agropecuarias, Universidad Nacional de Córdoba.

C.C. 509 - (5000) - Córdoba - ARGENTINA ranobile@agro.uncor.edu 